\title{
Do reference surfaces influence exocentric pointing?
}

\author{
M.J.A. Doumen, A.M.L. Kappers *, J.J. Koenderink \\ Universiteit Utrecht, Helmholtz Instituut, Physics of Man, Princetonplein 5, 3584 CC Utrecht, The Netherlands
}

\section{A R T I C L E I N F O}

\section{Article history:}

Received 25 June 2007

Received in revised form 3 March 2008

Accepted 4 March 2008

\section{PsycINFO classification:}

2323

\section{Keywords:}

Visual perception

Depth cues

Visual space

Depth perception

Exocentric direction

\begin{abstract}
A B S T R A C T
All elements of the visual field are known to influence the perception of the egocentric distances of objects. Not only the ground surface of a scene, but also the surface at the back or other objects in the scene can affect an observer's egocentric distance estimation of an object. We tested whether this is also true for exocentric direction estimations. We used an exocentric pointing task to test whether the presence of poster-boards in the visual scene would influence the perception of the exocentric direction between two test-objects. In this task the observer has to direct a pointer, with a remote control, to a target. We placed the poster-boards at various positions in the visual field to test whether these boards would affect the settings of the observer. We found that they only affected the settings when they directly served as a reference for orienting the pointer to the target.
\end{abstract}

(c) 2008 Elsevier B.V. All rights reserved.

\section{Introduction}

In 1950, Gibson proposed his ground theory, according to which the ground surface of a scene serves as a reference for perceiving the spatial layout of a scene. Nowadays, this idea is still very influential in the area of depth perception. Many scientists have tested the ground theory and developed it further. For example, Sinai, Ooi, and He (1998) concluded that a homogeneous ground surface is important for egocentric distance estimation. If the surface contains a discontinuous texture, the distance is underestimated (Feria, Braunstein, \& Andersen, 2003). Furthermore, the egocentric distance is underestimated when there is a gap in the surface between observer and test-object or when an occluder is conceiling part of this surface (He, Wu, Ooi, Yarbrough, \& Wu, 2004). These problems with distance estimation across a non-continuous ground surface arise because people may use a 'surface integration' method to estimate egocentric distances by estimating piece by piece the size of patches of the ground surface from close to the observer to the object ( $\mathrm{Wu}$, Ooi, \& He, 2004).

A few scientists have wondered whether the ground surface is a special surface for depth perception or whether every available surface in the visual scene is as important as reference as this ground surface. For example, Bian, Braunstein, and Andersen (2005) concluded that the ground surface is a special case. Objects

\footnotetext{
* Corresponding author. Tel.: +31 30253 2834; fax: +31 302522664.

E-mail address: a.m.l.kappers@phys.uu.nl (A.M.L. Kappers).
}

that could be interpreted as hanging from the ceiling or standing on the floor were perceived as standing on the ground. This is probably a very reasonable assumption since most objects in our visual world tend to be standing on a surface rather than hanging from it.

Another important surface could be the surface behind the testobjects (Glennerster \& McKee, 1999). For example, He and Ooi (2000) found that a vertical surface tends to be perceived as more frontoparallel than it actually is. Thus, a surface that is slanted around a vertical axis behind test-objects will affect the exocentric direction between these objects.

There are often numerous objects in the visual scene besides the reference surfaces discussed above. An important, but not often quantitatively investigated question is whether the presence of one object will influence the perception of the position of another. Daniels and Gordon (1993) investigated this idea. They used a 3D Poggendorff illusion. Two obliquely oriented vertical surfaces placed at eye-height of the observer had to be aligned. These surfaces were rotated $45^{\circ}$ from the frontoparallel plane of the observer. The observers had to align these two surfaces by shifting the position of the more distant surface along the mid-sagittal plane. The more distant surface was placed too close to the observer to be collinear to the other surface. Thus, a shift towards equidistance was found in this case. This shift was enlarged when a frontoparallel surface was placed in front and between the two test-surfaces. Daniels and Gordon (1993) concluded that the intrinsic bias (equidistance tendency) was enlarged because less information was present, i.e. the ground surface between the observer and the 
two test-objects was not visible to the observer in its entirety. In other words, when less information is available to estimate an egocentric distance, the more observers rely on intrinsic biases. This is an idea that has been proposed by many authors including Wagner (1985).

However, the presence of objects does not need to disturb the perception of the distances of other objects. It might well facilitate the perception of positions of other objects by giving extra references via nested contacts with the ground surface (Meng \& Sedgewick, 2001, 2002) or by providing extra depth information via occlusion, relative size or other depth cues.

Most work described above concerns egocentric distance estimations. However, we wondered how objects in the visual field influence the perception of exocentric direction. In our previous work, we used an exocentric pointing task in which we instructed the observer to orient a pointer to let it point towards a ball, clearly an exocentric direction estimation task. In these 2D experiments (Doumen, Kappers, \& Koenderink, 2005) both the pointer and the ball were positioned on the floor. The observers could make use of this information by extrapolating the information provided by the floor up to eye-level (the height at which the ball and the pointer were placed). However, in our 3D work (Doumen, Kappers, \& Koenderink, 2006) the pointer was positioned on the floor, whereas the ball was suspended from the ceiling. Although the spread of the data was somewhat larger in this 3D experiment, the size and the pattern of the deviations were comparable. This increase in spread might reflect an increase in difficulty experienced by the observers. An increase in the difficulty level of the task might reflect the change from having two objects connected to the floor to having only one of the objects connected to the floor. In the latter case, the observers might deduce the position at which the ball would be standing on the floor and then estimate the exocentric direction with reference to the ground surface. This deduction could lead to more variable data. However, this increase in difficulty level might also simply reflect the transition from a 2D to a 3D task, i.e. an extra degree of freedom could give rise to greater uncertainty of the observers.

In the current study, we investigated whether objects in the visual scene could facilitate the perception of the relative positions of two other objects. We did these experiments in an indoor setting. The square room had white walls, a floor with a texture on it and a ceiling that was covered with a white iron grid from which the balls were suspended. Points were marked on the floor to position the pointer. All intersections between walls, floor and ceiling were visible, as well as the positions where the pointer touched the floor and where the ball was suspended from the iron grid at the ceiling of the room. We placed poster-boards in the visual scene at different positions for different experiments. Placing a poster-board in between the observer and the two objects would probably result in less accurate settings because the space between observer and objects is not entirely visible. The ground surface in particular is partly occluded. The occlusion of the ground surface concerns the area between the pointer and the ball, contrary to the experiments of He and colleagues (2004), where the area between the observer and the stimuli was occluded from view. We chose to occlude the ground surface between the pointer and the ball because in contrast to egocentric distance estimations, for exocentric direction estimations, the relative positions of the two objects contain more important information than the position of the observer relative to the objects. If the poster-boards are placed behind the two objects or in between, the part of the ground surface that is needed for the estimation will be totally visible and, therefore, no problems will arise in this condition. However, when the poster-board is placed across the line between the two objects, this might confuse the observer. Furthermore, we believe that extra objects around the testobjects will only help the observers by providing extra references.

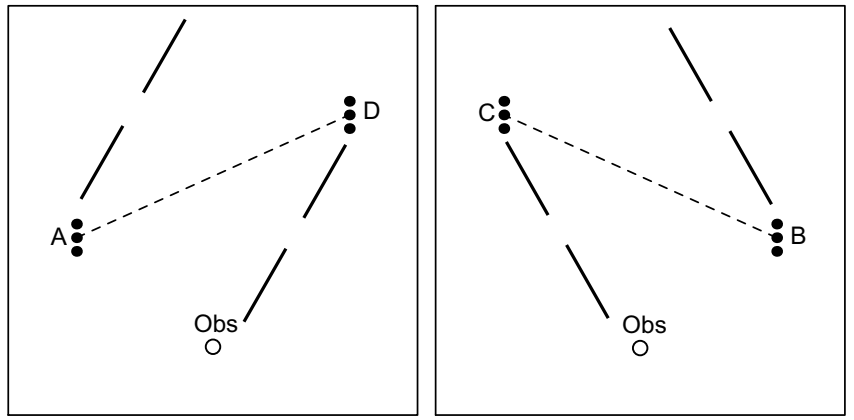

Fig. 1. Top-view of the experimental room of Experiment 3. The circle represents the position of the observer, the dots the positions of the pointer and the ball. The dashed lines give the veridical pointing-direction when the observer is pointing to the middle ball. The solid lines represent the positions of the poster-boards.

Placing objects at random positions in the room might not be sufficient, but placing them at positions that restrict angles etc. might reduce the deviations. For example, poster-boards placed on a line extending from the pointer's position provide extra information. In the case of Fig. 1, when pointing from A to D the observer knows that the pointer should be directed at the right side of the boards. In this circumstance, the poster-boards restrict the pointing angle to a substantial degree.

Thus, we conducted three experiments in which we placed poster-boards at different locations relative to the two test-objects and looked at the effects it had on the settings of the observers. Depending on the positions of the boards, the effect might be facilitating or even disturbing.

\section{General methods}

\subsection{Observers}

Undergraduate students, who were paid for their efforts, participated in the experiments described below. They had little or no experience in psychophysical experiments and were naive as to the goals and the purposes of the experiments. The observers did not participate in more than one of the experiments described below. They all had normal or corrected-to-normal visual acuity. Before the start of the experiment, each observer was tested for stereovision. They all had a stereo-acuity of 60 ", or better.

\subsection{Experimental set-up}

The experimental room measured $6 \mathrm{~m}$ by $6 \mathrm{~m}$. The wall opposite to the observer was white, with some electrical sockets near the floor. The wall on the left-hand side of the observer contained four blinded windows with radiators below them. The wall on the right-hand side contained two grey doors. Markers were placed on the floor to mark the positions of the objects that were used in the experiments. From the ceiling a horizontal iron grid was suspended ( $3 \mathrm{~m}$ above the floor). Attached to this grid were some white cubes that were used for other experiments. The cubes measured $20 \mathrm{~cm} \times 30 \mathrm{~cm} \times 15 \mathrm{~cm}$ and did not interfere with the stimuli in the visual field of the observer. From the iron grid hung a green ball $1.5 \mathrm{~m}$ above the ground. The ball had a diameter of $6 \mathrm{~cm}$ and could be placed at different positions. The pointer we used for this experiment consisted of a horizontal green rod ( $25 \mathrm{~cm}$ long and $2 \mathrm{~cm}$ thick, with one sharp endpoint) bisected by a yellow disk (diameter $8.2 \mathrm{~cm}$ and thickness $1 \mathrm{~cm}$ ) that was perpendicular to the rod. The rod passed through the centre of the disk and the disk was attached to a vertical iron rod so that the height of the rod was $1.5 \mathrm{~m}$. The foot of the pointer contained 
the motor that rotated the pointer and a protractor that was used to read the settings of the observer. A screen in front of the foot prevented the observer from seeing the protractor and the square shape of the foot. The observer could rotate the pointer using a remote control. The observer was seated on a revolving chair that could be adjusted in height so that the eye-height of each observer was at $1.5 \mathrm{~m}$. In the experimental conditions described below, we placed poster-boards in the experimental room. These posterboards measured $200 \times 90 \mathrm{~cm}(h \times w)$; each board was covered with equal sized posters with a brick-wall-pattern on them. The posters were used to help the observer to see the orientation of the poster-boards clearly.

\subsection{Procedure}

In all experiments described below, a similar procedure was followed. The observer sat on the revolving chair and the chair-height was adjusted. The experimenter explained the task: with the sharp endpoint of the rod, point to the ball as accurately as possible. The observer was instructed to remain seated and not to move his upper body. He was allowed to rotate his head and the chair. The observer always had a practice trial before starting the real experiment. After each trial, the observer had to close his eyes so that he could not see the experimenter reading the protractor and rearranging the objects for the next trial. The observer was also asked to rotate the pointer a little before opening his eyes again. This ensured that he did not have information about his previous setting. Each experiment took approximately 30 min for each observer.

\subsection{Analysis}

The dependent variable of the experiments discussed below can have a negative or a positive value. The directions of the deviations most often found (Doumen et al., 2005) are illustrated in Fig. 2: an overshoot when the pointer is closer to the observer than the target (Fig. 2A) and an undershoot when the target is closer to the observer (Fig. 2B). In our previous work, overshoots were defined as positive deviations and undershoots as negative. However, in the present experiments, we are not interested in the direction of the deviations, but only in the size of the deviations. Therefore, we defined the deviations in the expected direction as positive; this definition makes the analysis easier and the subsequent figures more readily interpretable. Thus, in the present experiments, a positive value means that the observer directed the pointer in the directions as depicted in Fig. 2. If the deviations are negative, the obser-
A

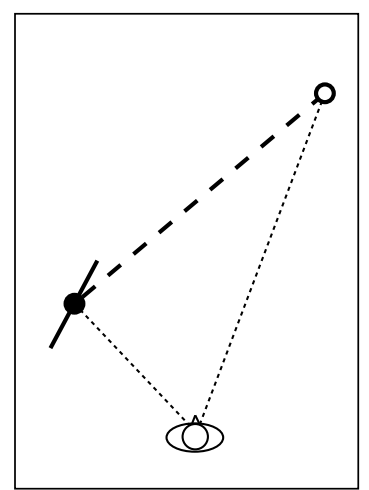

B

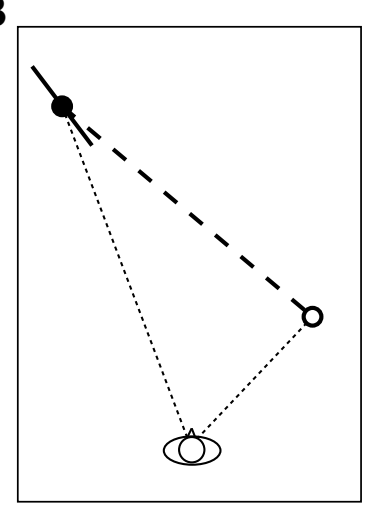

Fig. 2. Example of settings for the pointing task. The dot with line represents the pointer, the circle the ball, the thick dashed line the veridical pointing-direction and the thin dashed line the lines from observer to the pointer and the ball. (A) Forward pointing, (B) backward pointing. In both examples, the deviations are positive. ver did set the pointer in a direction on the other side of the dashed line (an undershoot in case the pointer is closer to the observer and an overshoot in case the target is closer to the observer).

In the experiments described below, we conducted analyses of variance with repeated measures. In the first experiment we added contrast analyses to enable us to look at the data in more detail: in the last experiments, we did this with a few additional $t$-tests.

\section{Experiment 1: poster-boards in between the pointer and ball}

In Experiment 1, a poster-board was always placed between the pointer and the ball in the visual field at places that were thought to affect the settings of the observers. More specifically, the posterboard was placed on the line dividing the room into two symmetrical halves through the mid-sagittal plane of the observer (when looking straight ahead at the opposite wall). The poster-board was placed perpendicular to the mid-sagittal plane at three different distances from the observer: closer to the observer than the pointer and ball (position 1 in Fig. 3), between the pointer and the ball (the veridical pointing-direction was through the posterboard, position 2 in Fig. 3) and further away from the observer than the pointer and the ball (position 3 in Fig. 3). In all these positions, the poster-boards blocked the view of the intersection between the floor and the back-wall between the pointer and the ball. Furthermore, when the poster-board was close to the observer or in the middle position, the line between the pointer and the ball was blocked from the view. Thus, if observers do the task by following this line, they will be distracted in these two conditions. On the other hand, the presence of these poster-boards as objects connected to the floor might give the observer extra information about the positions of the pointer and the ball.

\subsection{Methods}

For Experiment 1 five naive female undergraduate students participated as observers. If the observer is positioned at the origin of a Cartesian coordinate system, the pointer could be either at positions $\mathrm{A}(-140,220)$ or $\mathrm{C}(-210,330)$ and the ball at positions $\mathrm{B}$

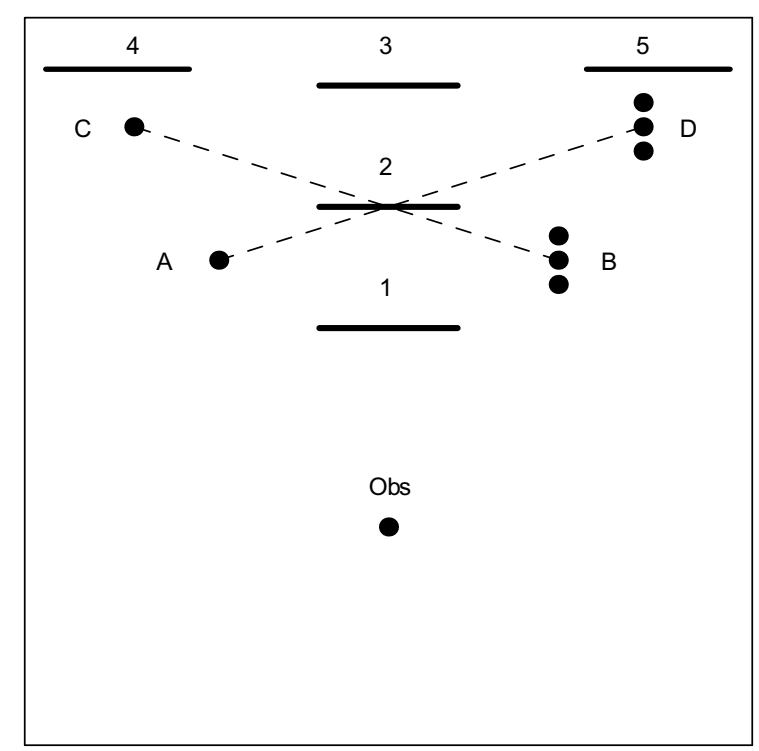

Fig. 3. Top-view of the experimental room of Experiments 1 and 2. The square represents the wall of the experimental room, the dots the positions of the observer (Obs), the pointer ( $A$ and $C$ ) and the ball ( $B$ and $D)$. The dashed lines represent the veridical pointing-directions (when the observer is pointing to the middle ball) and the solid lines represent the positions of the poster-boards (1, 2 and 3 for Experiment 1, 4 and 5 for Experiment 2). 
$(140,220)$ or $\mathrm{D}(210,330)$ in $\mathrm{cm}$ as depicted in Fig. 3. This means that the two objects always had a separation angle of $65^{\circ}$ and were at a distance of 260 or $390 \mathrm{~cm}$ from the observer. This is depicted in Fig. 3. In this figure, the square represents the walls of the experimental room, the dots represent the positions of the observer, pointer and ball, and the dashed lines give the pointing-directions. The balls could be at the given position or either $20 \mathrm{~cm}$ closer to or further away from the observer. This means that for position $B$ the ball could be at the following positions: $(140,200),(140,220)$ or $(140,240)$. The difference was large enough for the observer to notice the difference between the positions, but small enough not to change the relative distance too much. We did this to make sure that the observer could not remember the orientation of the pointer in the given situations. The poster-boards were placed on the mid-sagittal plane oriented frontoparallel for the observer, at distances of 164, 264 and $364 \mathrm{~cm}$ from the observer. These positions are depicted in Fig. 3 as the solid lines with numbers 1-3.

The observers always had to point from point $A$ to $D$ and from point $C$ to $B$. This was done for conditions with poster-boards (the three different experimental conditions) and without posterboards. Thus, Experiment 1 consisted of $2 \times 3 \times 4=24$ trials (\# pointer-position, \# ball-positions, \# poster-board conditions). We performed a repeated measures analysis, together with a contrast analysis. We added the contrast analysis for the variable posterboards because it allows us to investigate the effect of the three experimental conditions in contrast to the control condition without the poster-boards.

\subsection{Results of Experiment 1}

Typical settings of observers are shown in Fig. 2. What can be seen in these data is that when the pointer was closer to the observer than the ball, most observers directed the pointer further away than veridical, whereas when the pointer was positioned further away from the observer than the ball they directed the pointer between themselves and the ball. Fig. 4 gives the mean deviations of all observers for Experiment 1. The grey bars represent the trials in which the observer pointed from A to $D$, the black bars when the observer pointed from $C$ to $B$. The first two bars represent the control condition, without the poster-boards. The other groups of bars represent the conditions with poster-boards: boards closer to, in the middle and further away from the observer, respectively. The error-bars represent the standard errors of the mean. The data in Fig. 4 show only positive deviations, which means that all observers pointed as shown in Fig. 2. The most striking point in this figure is the difference between the sizes of the deviations when observers pointed from $A$ to $D$ and from $C$ to $B$. However, there seemed to be no difference between the different poster-board conditions. We conducted an analysis of variance with repeated measures on these data. The deviation is the dependent variable, whereas the pointing-direction (forward versus backward pointing) and the presence of poster-boards were the independent variables. Although we found a difference between pointing forward or backward $(F(1,4)=27.500, p=0.006)$, we found no overall effect of the different poster-board conditions $(F(1,4)=1.490, p=0.426)$. The interaction-effect between pointing-direction and the presence of poster-boards did not reach significance $(F(1,4)=7.070$, $p=0.126$ ). Furthermore, a contrast analysis on the different poster-board conditions did not reveal any effect of the three posterboards conditions when compared to the control condition.

\section{Experiment 2: poster-boards behind the pointer and the ball}

A second experiment was conducted with poster-boards in the visual field of the observer. The poster-boards were placed behind the pointer and the ball. Thus, in the second experiment, the poster-boards served as a background against which the pointer and the ball were presented to the observer. The poster-boards were never between the observer, pointer and ball. Thus, the floor between the objects and the observer was always visible to the observer. The poster-boards, however, blocked the view of the corners of the room in the experimental conditions. If the observers used the geometry of the room when doing the task, they may not have sufficient information to do the task in the experimental condition. This could be visible in the signed errors or in the spread of the data.

However, since these poster-boards are objects connected to the floor, they may give the observer extra information about the positions of the pointer and the ball.

Two possible hypotheses are given for this experiment: first, the poster-boards block the view of the corners of the room (intersections of the floor and two walls), thereby reducing the information about the geometry of the room present in the visual scene and increasing the size or the spread of the deviations. Second, the positions of the poster-boards could give the observer extra information about the relative positions of the pointer and the ball, thereby reducing the size or the spread of the deviations.

\subsection{Method}

Five naive undergraduate students (three male and two female) participated as observers in Experiment 2. The poster-boards were placed $50 \mathrm{~cm}$ behind the pointer and the ball in such a way that the pointer and the ball were projected onto the middle of the board as

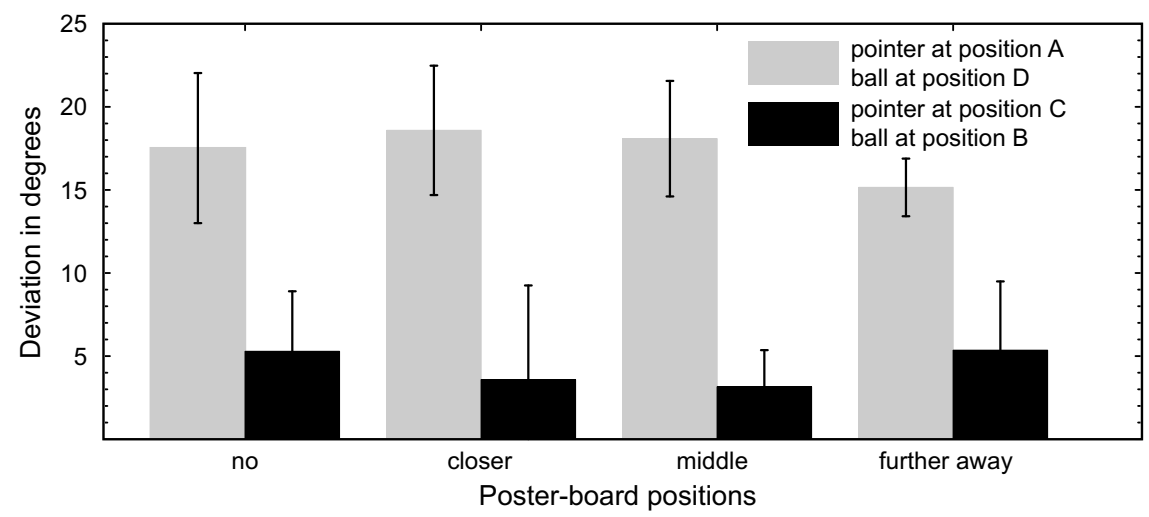

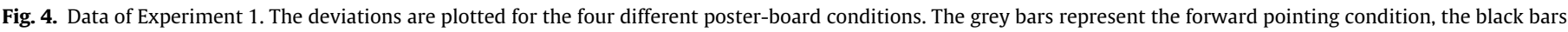
represent the backward pointing condition. The error-bars give the standard errors of the mean. 


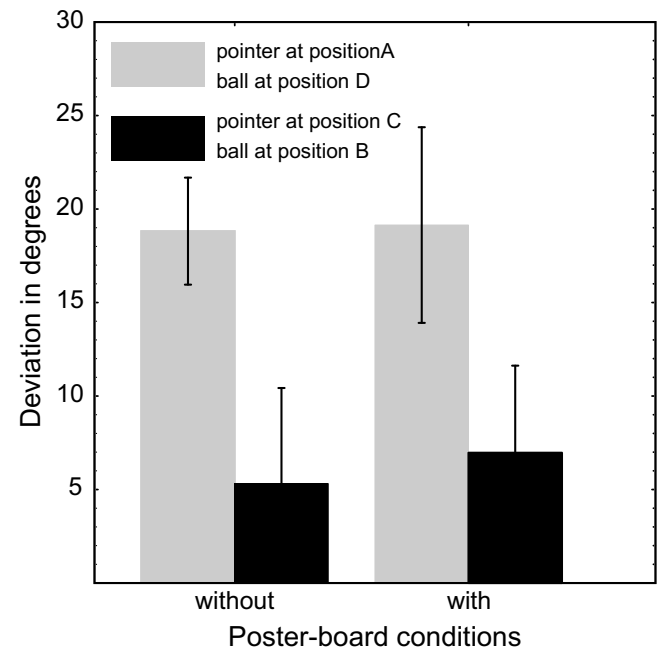

Fig. 5. Data of Experiment 2. The deviations are plotted for the two different posterboard conditions. The grey bars represent the forward pointing condition, the black bars represent the backward pointing condition. The error-bars give the standard errors of the mean.

seen from the observer's point of view (Fig. 3, solid lines 4 and 5). We had four different prints attached to the poster-boards, and a control condition without poster-boards. Since the results did not depend in any systematic way on the poster-board prints, we leave this parameter out of the further analyses.

As in Experiment 1, the observers always had to point from point $A$ to $D$ and from point $C$ to $B$. This was done for conditions with poster-boards (four different experimental conditions, prints) and without poster-boards. Thus, Experiment 2 consisted of $2 \times 3 \times 5=30$ trials (\# pointer-position, \# ball-positions, \# poster-board conditions $(4+1)$ ). We performed a repeated measures analysis, together with a contrast analysis. We added the contrast analysis for the variable poster-boards because it allows us to investigate the effect of the four experimental conditions (that are not addressed in detail) in contrast to the control condition without the poster-boards.

\subsection{Results of Experiment 2}

The data of Experiment 2 are plotted in Fig. 5. The bars represent the same variables as described above. However, the second group of bars represents the mean of the settings for the four different prints. Again, we can see that there is only a difference between forward versus backward pointing but not between the two poster-board conditions. The analysis of variance (with repeated measures and contrast analysis) that we conducted on these data again revealed an effect of pointing-direction $(F(1,4)=43.212, p=0.003)$ but neither the effect of the posterboard conditions $(F(1,4)=1.171, p=0.360)$ nor an interaction-effect $(F(1,4)=0.467, p=0.759)$. Furthermore, the contrast analysis comparing the deviations for the four different prints to the control condition revealed no effect of the poster-boards.

\section{Experiment 3: poster-boards restricting the pointing angle}

So far, we have seen that the presence of the poster-boards in the visual field did not have any effect on the settings of the observers. We did not find an increase or decrease in signed deviations nor did we find an increase or decrease in the spread of the data. Thus, the mere presence of poster-boards in the visual field did not affect the settings of the observers. However, we see that observers show a highly regular pattern of deviations, with only deviations in directions as shown in Fig. 2. Furthermore, when the pointer is closer to the observer than the ball (Fig. 2A), the deviations are larger than when the pointer is further away from the observer (Fig. 2B). It could be that in the situation of Fig. $2 \mathrm{~B}$ the observer's own body-position serves as a reference for orienting the pointer. In other words, the pointing angle is restricted by the location of the observer. Thus, in the third experiment we placed poster-boards at positions that restricted the pointing angle in the pointing-directions as depicted in Fig. 2. The solid lines in Fig. 1 represent the position of the poster-boards. The boards extend from positions $C$ and $D$ to the position of the observer, and from positions A and B away from the observer. In these experimental conditions, the pointing angle is restricted since the observer knows that if, for example, the pointer is at position $A$, the direction of pointing is to the right of the poster-boards. This pointing angle for pointer-positions $C$ and $D$ is already restricted by the observer's own body-position without the presence of posterboards. Thus, in the experimental condition, we expect to find a reduction in deviations when the pointer is closer to the observer. For example, when the pointer is at position A and the ball at position D (Fig. 1) we expect to find smaller deviations when the poster-boards are present than when the poster-boards are not present in the scene. However, no reduction in deviations are expected for the experimental condition when the ball is closer to the observer. For instance, when the pointer is at position D and the ball at position A (Fig. 1), we do not expect to find smaller deviations when the poster-boards are present than when they are not present. A reduction in deviations is not expected since the poster-boards do not contribute to the reduction of the pointing angle in this situation. It could be that the difference in the size of the deviations between situations A and B of Fig. 2 will disappear since the pointing angle is restricted to the same degree in both situations.

\subsection{Methods}

Six male and two female observers participated in this experiment.

The experimental set-up consisted of the pointer, ball, four poster-boards and a revolving chair. The coordinates (in $\mathrm{cm}$ ) of the positions of these objects were as follows: the observer was placed at $(0,0)$, the pointer and the ball could be either at positions A $(-200,160), B(200,160), C(-200,340)$ or D $(200,340)$ as depicted in the two panels of Fig. 1. Both the pointer and the ball could be at positions A through $\mathrm{D}$ (four pointer and ball-positions). This results in the parameter pointing-direction (pointing from left to right and vice versa) and forwards versus backwards pointing. In addition to an experimental condition with the poster-boards we had a control condition without the boards (two experimental conditions). We varied the positions of the ball to prevent the observer from guiding his settings by using the information provided by his view of the pointer (three repetitions/ball-positions). The difference between the positions of the ball was $20 \mathrm{~cm}$, as is explained in the Methods section of Experiments 1 and 2. This resulted in $4 \times 2 \times 3=24$ trials (\# pointer \& ball combinations, \# experimental conditions, \# repetitions/ball-positions).

\subsection{Results}

The data are plotted in Fig. 6. Each bar represents the mean of the values for all eight observers. The grey bars represent the data for the control condition, i.e. without the poster-boards. The black bars represent the data for the experimental condition, i.e. with the poster-boards. Each group of bars represents the results for one 


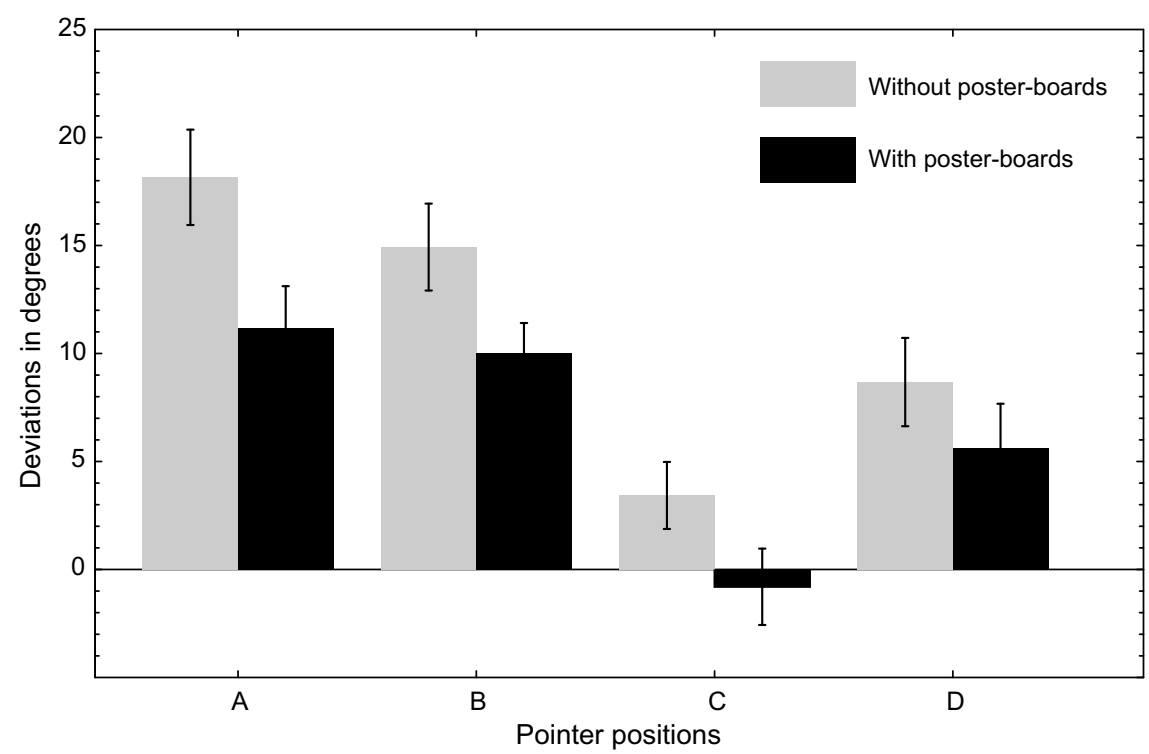

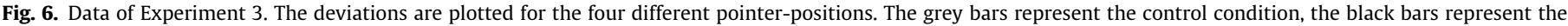
condition with the poster-boards. The error-bars give the standard errors of the mean, the mean variance between observers.

position of the pointer. The first two groups (A and B) are the cases in which the pointing-direction is forward; the second two groups of bars ( $C$ and $D$ ) represent the cases in which the pointing-direction is backward.

The first thing to notice is that most deviations are positive and the deviations for pointing forward (A and B) are larger than for pointing backward ( $C$ and $D)$. Furthermore, the deviations are larger in the conditions without poster-boards than in the conditions with poster-boards.

We conducted an analysis of variance with repeated measures on these data. The dependent variables were the presence of poster-boards, pointing forward or backward and the direction of pointing (from left to right or vice versa). There was a significant effect of both the presence of the poster-boards $(F(2,7)=18.396$, $p=0.004$ ) and forward versus backward pointing (A, B versus $C$,
D; $F(2,7)=45.285, p<0.001)$. There was a significant interaction between the right and left pointing-directions (A, C versus $B, D$ ) and forward versus backward pointing (A, B versus $C, D)$, as can be seen in Fig. $6(F(2,7)=43.753, p<0.001)$.

However, for questions concerning the nature of the effect of the poster-boards on the settings of the observers, this analysis does not provide direct answers. Therefore, we conducted three paired $t$-tests on these data $(\alpha=0.0125(0.05 / 4))$. We found a difference between pointing forward and backward (A, B versus C, D for both experimental conditions (without boards: $t(7)=6.925$, $p<0.001$, with boards: $t(7)=5.411, p=0.001)$. Furthermore, we found a significant difference between the two experimental conditions (with versus without poster-boards) when the pointer was closer to the observer than the ball $(t(7)=4.825, p=0.002)$. However, the difference between the experimental and the control

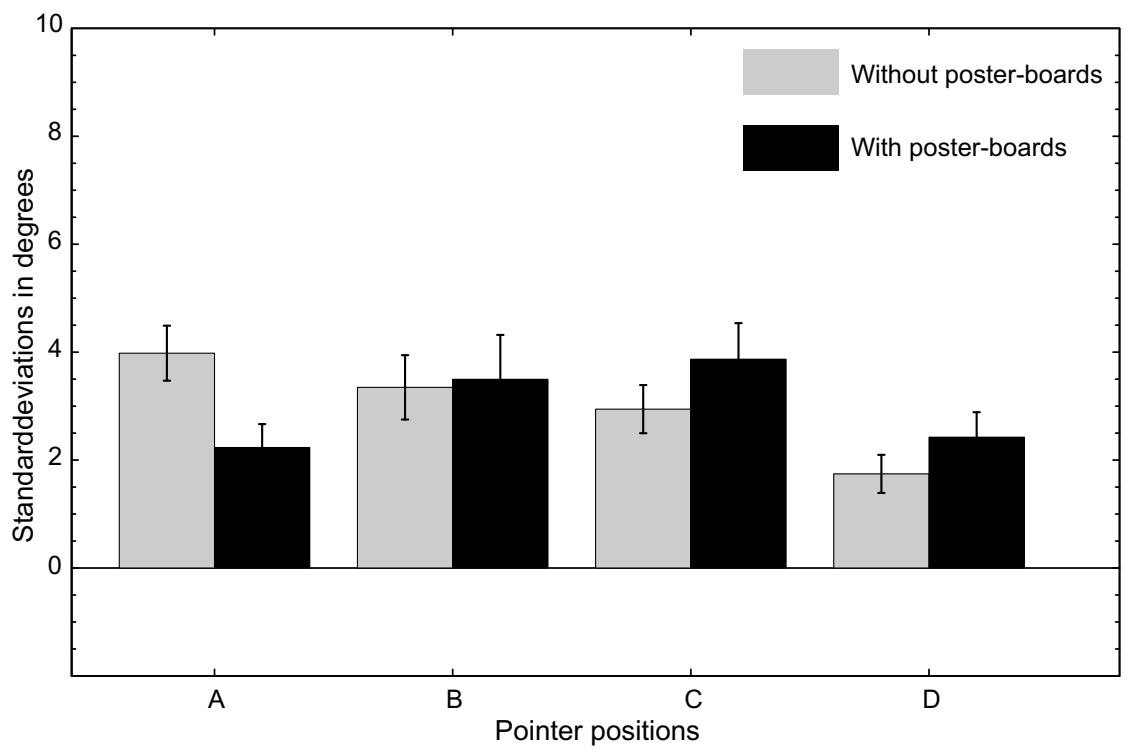

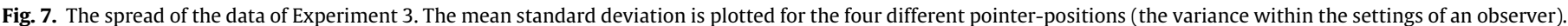

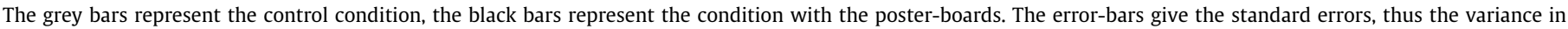
standard deviations between observers. 
condition when the pointer was further away from the observer than the ball was not significant $(t(7)=2.781, p=0.027)$.

In Fig. 7 the standard deviations of the settings are plotted against the pointer-positions. Thus, the value is the mean of the measured standard deviations found for each observer. As in Fig. 6, the grey bars represent the trials without poster-boards, whereas the black bars represent the trials with poster-boards. The error bars represent the variation between observers, measured in standard error of the mean. In Fig. 7, the bars do not show a structural pattern so we can conclude that the presence of the poster-boards does not have an effect on the spread of the data.

\section{Discussion and conclusions}

The effect of the difference in the direction of the deviations when the pointer is close to the observer or far away, which we found in our earlier work and in the work of Cuijpers and colleagues (Cuijpers, Kappers, \& Koenderink, 2000; Doumen et al., $2005,2006)$, is replicated in the present work. The observer overshoots the ball. Furthermore, we replicated the asymmetry between forward and backward pointing in the size of the deviations.

From the first two experiments, we can conclude that the mere presence of the poster-boards did not result in any effect on the pointer-settings of the observers. Even the occlusion of the parts of the ground surface (Experiment 1) did not affect our data. This is unexpected since we hypothesized that the occlusion of the ground surface would affect an exocentric direction judgment because the occlusion of the ground surface between the observer and the stimulus does affect an egocentric distance estimation (Daniels \& Gordon, 1993; He, Wu, Ooi, Yarbrough, \& Wu, 2004; Sinai et al., 1998; Wu et al., 2004). This might be explained by the fact that in the present experiments, the pointer was positioned on the floor, whereas the ball was suspended from the ceiling. This might produce a situation in which the ground surface is less important than in the experiments described in the introduction. It could also be that the poster-boards did not occlude the primary features of the experimental room that are crucial for the geometry of the room if it is to be a good reference for the observer. Thus, a more efficient way of occluding edges etcetera might induce larger deviations in the pointing task. However, it could also mean that observers do not use the information given by the geometry of the room. This information might be too indirect to be useful for the observer. Furthermore, the fact that the "pointing-pathway" between the pointer and the ball was blocked from the view did not affect our data. Thus, it is not important to be able to follow this path with one's eyes.

An alternative explanation could be that facilitatory effects cancel out the restricting effects and thereby produce no effect at all. However, since neither Experiment 1 nor Experiment 2 produces any effects and these experiments concern completely different positions of the poster-boards, we believe it is highly unlikely that they would produce effects that completely cancel out. For the present, we will conclude that no effects of the presence of poster-boards were measured in Experiments 1 and 2.

As discussed above, the mere presence of the poster-boards does not give more cues or information for seeing depth. It does not facilitate the perception of the locations of objects, as can be seen in Experiments 1 and 2. However, this is dependent on the exact positions of the poster-boards. In the last experiment, we restricted the pointing angle with poster-boards. In the condition with the poster-boards we find significantly smaller deviations than in the condition without the poster-boards. So, we can conclude that the observers had a more veridical estimation of the relative positions of the pointer and the ball. They can use the poster-boards as a reference for orienting the pointer. We found a significant difference between the condition with posterboards and without poster-boards when the pointer was close to the observer, but not when the ball was close to the observer. Although a difference is visible in Fig. 6 when the ball is close to the observer, this difference does not reach significance.

We could not find existing theories that can explain this effect. However, a simple explanation could be that the poster-boards provide extra depth cues that are useful for the observer to estimate the relative positions of the two objects. For example, the poster-boards give plenty of information about depth in the ground surface due to linear perspective and the fact that the poster-boards touch the floor. Furthermore, the poster-boards are aligned in a plane that clearly (due to depth cues described above) overshoots the direction of the ball. Therefore, more information is present in the poster-board condition than in the control condition. Thereby, the observers can rely more on the contextual information and are less influenced by intrinsic biases as described by Wagner (1985), and Daniels and Gordon (1993).

Besides serving as a reference for judging the exocentric direction, the poster-boards could affect the perception of the slant of the pointer. He and Ooi (2000) found observers to judge vertical planes as positioned more frontoparallel than they actually were. When we extrapolate this knowledge to our experiments, the reference planes (poster-boards) will be perceived as more frontoparallel and possibly the observer perceives the pointer more frontoparallel than it was objectively oriented. However, when the poster-boards are perceived more frontoparallel, the perceived restriction of the pointing angle is even larger than the actual restriction. Consequently, these effects will be counterbalanced.

As mentioned above, in the backward pointing condition (Fig. 2B) we find smaller deviations than in the forward pointing condition (Fig. 2A). We theorized that this could be due to a restriction of the pointing angle with the observers' own body-position. As can be seen in Fig. 2B, the typical setting of an observer is orienting the pointer in a direction in between the veridical orientation and the line between the pointer and the observer himself. Since humans are very sensitive to being pointed at, an observer will never orient the pointer more towards himself than the ball. Therefore, an extra external reference is present in this situation which results in a less influence of intrinsic biases. This results in more veridical settings than when the pointer is closer to the observer (Fig. 2A). In the poster-board condition of Experiment 3 we simulated this situation with the poster-boards restricting the pointing angle in both situations (Fig. $2 \mathrm{~A}$ and $\mathrm{B}$ ) to the same degree. When the pointer is far away from the observer, the small difference between experimental and control condition could be explained by the fact that the pointing angle was already restricted in the control condition. Thus, the restriction of the pointing angle seems to be an important cue for performance in a pointing task, whether this is due to the observer's own body-position or to other objects in the visual scene.

In summary, the presence of objects in the visual field of the observer does affect only the judgment of relative positions when they serve as a direct reference.

\section{Acknowledgements}

The investigations were supported by the Research Council for Earth and Life Sciences (ALW) with financial aid from the Netherlands Organization for Scientific Research (NWO).

\section{References}

Bian, Z., Braunstein, M. L., \& Andersen, G. L. (2005). The ground dominance effect in the perception of 3-d layout. Perception E' Psychophysics, 67, 802-815. 
Cuijpers, R. H., Kappers, A. M. L., \& Koenderink, J. J. (2000). Investigation of visual space using an exocentric pointing task. Perception $\mathcal{E}$ Psychophysics, 62 1556-1571.

Daniels, V., \& Gordon, I. E. (1993). Occlusion and the distortion of alignment in three-dimensional space. Perception, 22, 1037-1044.

Doumen, M. J. A., Kappers, A. M. L., \& Koenderink, J. J. (2005). Visual space under free viewing conditions. Perception \& Psychophysics, 67, 1177-1189.

Doumen, M. J. A., Kappers, A. M. L., \& Koenderink, J. J. (2006). Horizontal-vertical anisotropy in visual space. Acta Psychologica, 123, 219-239.

Feria, C. S., Braunstein, M. L., \& Andersen, G. L. (2003). Judging distance across texture discontinuities. Perception, 32, 1423-1440.

Gibson, J. J. (1950). The perception of the visual world. Cambridge: The Riverside Press.

Glennerster, A., \& McKee, S. P. (1999). Bias and sensitivity of stereo judgments in the presence of a slanted reference plane. Vision Research, 39, 3057-3069.
He, Z. J., \& Ooi, T. L. (2000). Perceiving binocular depth with reference to a common surface. Perception, 29, 1313-1334.

He, Z. J., Wu, B., Ooi, T. L., Yarbrough, G., \& Wu, J. (2004). Judging egocentric distances on the ground: Occlusion and surface integration. Perception, 33, 789-806.

Meng, J. C., \& Sedgewick, H. A. (2001). Distance perception mediated through nested contact relations among surfaces. Perception \& Psychophysics, 63, 1-15.

Meng, J. C., \& Sedgewick, H. A. (2002). Distance perception across spatial discontinuities. Perception E' Psychophysics, 64, 1-14.

Sinai, M. J., Ooi, T. L., \& He, Z. J. (1998). Terrain influences the accurate judgment of distance. Nature, 395, 497-500.

Wagner, M. (1985). The metric of visual space. Perception \& Psychophysics, 38, 483-495.

Wu, B., Ooi, T. L., \& He, Z. J. (2004). Perceiving distance accurately by a directional process of integrating ground information. Nature, 428, 73-77. 